\title{
Study on the Clarification Effect of Purple Sweet Potato, Jujube, Hawthorn Compound Juice Treated with Chitosan
}

\author{
Shulan Yu, Fang Zou, Wenjing Han and Xinwen Zhang \\ Weifang Vocational College, Weifang, Shandong 261031
}

\begin{abstract}
The clarification of purple sweet potato, jujube, hawthorn compound juice using chitosan is studied. The influence of the factors such as temperature, $\mathrm{pH}$ value, action time and dosage of chitosan on the compound juice clarification effect, is discussed. Through single factor experiments, the clarification process can be optimized and the best process parameters can be found by using multi-factor orthogonal experiments: the temperature is $50^{\circ} \mathrm{C}$, the $\mathrm{pH}=6.0$, the clarification time is $60 \mathrm{~min}$, and the chitosan dosage is $1.6 \mathrm{~g} / \mathrm{L}$. The results show that the clarification effect of chitosan for purple sweet potato, jujube, hawthorn compound juice is remarkable. And the light transmittance of compound juice can be over $96 \%$ after clarification.
\end{abstract}

Keywords: Purple sweet potato; Jujube; Hawthorn; Chitosan; Clarification

\section{壳聚糖对紫甘薯、大本、山楂 \\ 复合汁的澄清效果研究}

\author{
于淑兰，邹芳，韩文婧，张新文
}

(滩坊职业学院，山东 潍坊 261031）

摘要: 利用壳聚糖对紫甘薯、大本、山楂复合汁进行了澄清试验。探讨了温度、 $\mathrm{pH}$ 值、作用时间和壳聚糖用量等因素 对复合汁澄清效果的影响。通过单因素试验, 采用多因素正交试验对澄清工艺进行优化, 确定最优工艺参数为: 温度 $50^{\circ} \mathrm{C} 、$ $\mathrm{pH}$ 值为 6.0、作用时间 60min、壳聚糖用量 $1.6 \mathrm{~g} / \mathrm{L}$ 。实验结果表明, 壳聚糖澄清紫甘著、大本、山楂复合汁, 澄清效果好, 可使该复合汁的透光率达 $96 \%$ 以上。

关键词: 紫甘著; 大本; 山楂; 壳聚糖; 澄清

引言

紫甘薯又称紫番薯, 因含有丰富的花青素而使薯肉呈紫色至深紫色。紫甘薯除含有丰富的花青素外, 还含有植物蛋白、维生素、多糖、矿质元素等多种营养物质, 对人体具有较高营养价值, 是一种很好的保 健食品 [1]。近几年的研究表明, 紫甘薯花青素具有较强的抗氧化作用, 能够清除人体自由基, 可以起到 预防和治疗多种疾病。可见, 对紫甘薯深加工的研究, 有着广阔的发展前景。大隶是鼠李科植物, 果实中 含有蛋白质、脂肪、糖、抗坏血酸、钙、铁等多种人体所需的营养成分, 具有很高的药用价值。大東是一 种药食两用的食品, 大零中除含有丰富的多糖外, 还含有维生素 C、芦丁、皇苷、五环三萜类、环磷酸鸟 苷和环磷酸腺苷等物质, 具有提高机体免疫力和抗氧化作用。因此, 大苯是被人们公认的滋补佳品 $[2,3]$ 。 山楂是一种药食兼用的果品, 果实中含有丰富钙和维生素 C, 还含有蛋白质、脂肪、碳水化合物、山楂酸、 钙、磷、铁等矿物质等营养成分。除此之外, 山楂中还含有黄酮类和山萜类物质, 具有扩张血管及降血压 的作用 [4]。山楂还能够起到消食、健胃的作用。可见, 山楂具有较高的药用价值。综上所述, 对紫甘薯、 大苯、山楂等 3 种果蔬进行深加工, 有着重要现实意义和广阔的发展前景。 
壳聚糖主要是由节肢动物蟹、虾和昆虫等外壳中的甲壳素经脱乙酰化作用而制取的一种带正电荷的多 糖。根据文献报道 $[5,6]$, 壳聚糖分子所带的正电荷, 可与果疏汁中带负电荷的微粒相互作用, 形成絮凝 物而沉淀, 从而达到澄清的目的。目前, 我国果蔬汁的加工, 通常是采用酶法进行果蔬汁的澄清, 它存在 着操作时间长、成本高、澄清效果差等缺点。采用壳聚糖为澄清剂澄清果蔬汁, 具有澄清时间短、操作简 单、成本低、澄清效果好等优点 $[7,8]$ 。本文旨在采用壳聚糖为澄清剂, 对紫甘薯、大苯、山楂复合汁进 行澄清试验, 探讨壳聚糖在澄清该复合汁的过程中, 澄清温度、 $\mathrm{pH}$ 值、作用时间和壳聚糖用量等最优条件, 以期为紫甘薯、大本、山楂等的深加工提供参考依据。

\section{1 材料与方法}

\section{1 材料与设备}

新鲜紫甘薯（烟紫薯 2 号）、大東（金丝小本干果）、山楂（山楂干片）等均为市售; 壳聚糖 [9], 自制, 脱乙酰度 $86 \%$; $a$-淀粉酶: 邢台万达生物工程有限公司, 酶活力 $\geqslant 3700 \mathrm{u} / \mathrm{g}$; 糖化酶: 张家港金源 化工技术有限公司, 酶活力 $\geqslant 50000 \mathrm{u} / \mathrm{g}$ 。

电子分析天平; 数显恒温水浴锅; 1801 型紫外分光光度计; 酸度计; 打浆机; 离心机 (4000r/min); 不锈钢锅等。

\section{2 方法}

\subsection{1 澄清度测定方法}

复合汁澄清度的测定采用分光光度法。即以蒸馏水为参比, 在波长 $524 \mathrm{~nm}$ 处测定复合汁的透光率 ( T\%), $\mathrm{T}$ 值越大, 表明澄清度越高, 澄清效果越好。

\section{2.2 澄清方法[10]}

先将鲜紫甘薯蒸熟, 加水打浆, 先后加入 $a$-淀粉酶和糖化酶进行酶解得紫甘薯酶解液。再将大杳洗 净, 加一定量水加热, 冷却后打浆, 搅拌浸提, 过滤得春汁。然后, 把山楂干洗净, 加于一定量水, 加热 软化后进行打浆, 过滤得山楂汁。将制得的紫甘薯酶解液、春汁和山楂汁按一定比例混合, 得到混浊的复 合汁。最后, 将混浊的复合汁分别在不同的壳聚糖用量、温度、作用时间、 $\mathrm{pH}$ 等条件下进行澄清试验, 找 出各因素对澄清效果的影响。

\section{2 .3 复合汁澄清最优条件的确定方法}

在单因素试验的基础上，对复合汁澄清温度、pH值、作用时间和壳聚糖用量等因素进行正交试验，确 定最优工艺条件。

\section{3工艺流程}

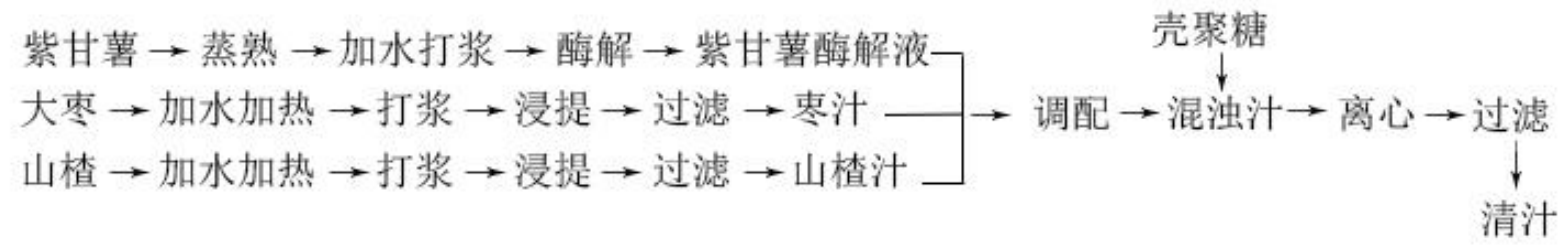

\section{4 操作要点}

1.4 .1 紫甘薯汁的制备

首先将新鲜紫甘薯洗净，切成 $3 \mathrm{~cm}$ 左右的薄片，加热煮沸 $15 \mathrm{~min}$, 然后，以 $1: 2$ 的料水比打浆; 再将 
$\mathrm{pH}$ 值调节为 6.0 ; 保持温度 $60^{\circ} \mathrm{C}$, 按每克干物质加入 3700 活力单位的 a -淀粉酶, 酶解 $40 \mathrm{~min}$; 然后, 将 $\mathrm{pH}$ 值调节为 4.3 , 按每克干物质加入 300 活力单位的糖化酶, 保持温度 $60^{\circ} \mathrm{C}$, 糖化 $60 \mathrm{~min}$; 最后加热至 $95^{\circ} \mathrm{C}$, 维持 30 秒进行灭酶, 得到紫甘薯汁。

\section{4.2 大東汁的制备}

将大東干果水洗干净后放入烘箱中, 在 $95^{\circ} \mathrm{C}$ 烘烤 $2 \mathrm{~h}$, 然后按料水比 $1: 6$ 的比例加入水, 在 $80^{\circ} \mathrm{C}$ 煮制 $1 \mathrm{~h}$, 最后打浆、过滤 (100目篮网) 得到大束汁。

1.4.3山楂汁的制备

将山楂干片按料水比 1: 7 的比例加入水, 加热到 $75^{\circ} \mathrm{C}$ 软化 1 小时, 打浆, 过滤 (100目篮网) 得到山 楂汁。

\section{4.4 复合汁的配制}

将紫甘薯汁、大東汁、山楂汁以不同比例进行复合配制，得到各组复合汁。以复合汁的口感、香气和 色泽等为评分标准, 进行综合评价, 找出复合汁的最佳配比。

\section{4 .5 杀菌}

将最佳配比的复合汁在 $85^{\circ} \mathrm{C}$ 条件下，加热杀菌 $15 \mathrm{~min}$ 。

\section{2 结果与分析}

\section{1 紫甘薯汁、大東汁及山楂汁配比对复合汁口感和风味的影响}

紫甘薯汁颜色紫红, 花青素含量高, 营养丰富, 但是, 味道一般; 大本汁具有浓郁的大菄香味, 营养 价值高, 是主要的口感和风味来源; 山楂汁味酸, 主要用来调节糖酸比, 使复合汁的口感更佳。因此, 综 合这些特点, 进行不同配比试验, 从中篮选出风味最佳的紫甘薯汁、大菄汁、山楂汁配比为: $30.0: 10.0: 10.0$ 。

\section{2 澄清剂（壳聚糖）用量对复合汁透光率的影响}

在复合汁 $\mathrm{pH}$ 为 6.0 , 温度 $60^{\circ} \mathrm{C}$, 澄清时间为 $60 \mathrm{~min}$ 的条件下, 壳聚糖用量对复合汁透光率的影响见图 1 。

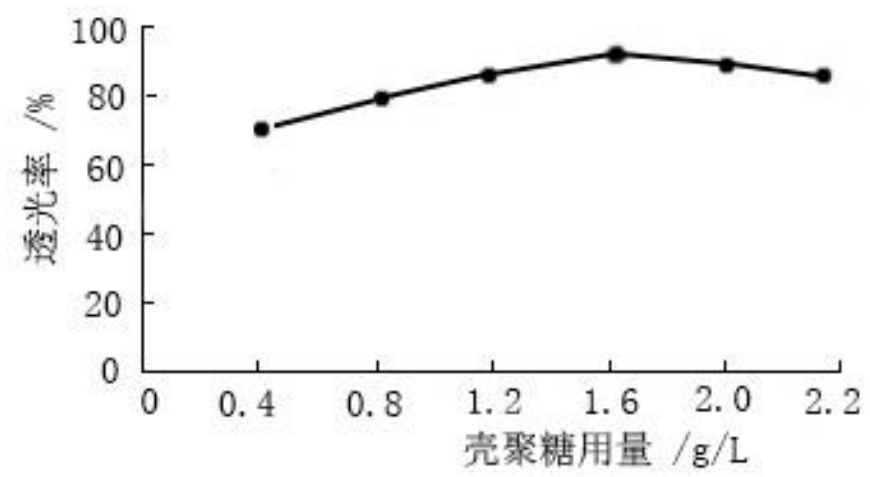

图 1 壳聚糖用量对复合汁透光率的影响

Figure 1. The influence of dosage of chitosan on the compound juice transmittance

由图1得知, 在壳聚糖用量较低时, 透光率随着壳聚糖用量的增加而增大, 当壳聚糖用量达到 $1.6 \mathrm{~g} / \mathrm{L}$ 时, 透光率达到最大值。壳聚糖用量大于 $1.6 \mathrm{~g} / \mathrm{L}$ 时, 透光率逐渐降低。 


\section{3 温度对复合汁透光率的影响}

在壳聚糖用量 $1.6 \mathrm{~g} / \mathrm{L}, \mathrm{pH}=6$, 作用时间 $60 \mathrm{~min}$ 的条件下, 温度与复合汁透光率关系图 2 。

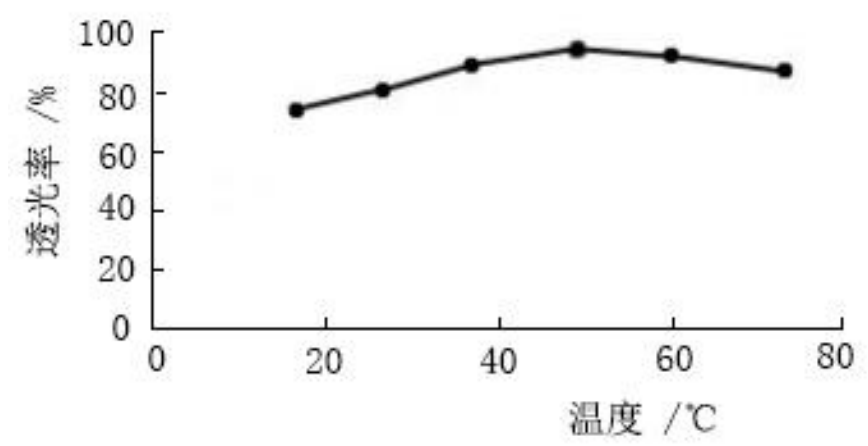

图 2 温度对复合汁透光率的影响

Figure 2. The influence of temperature on complex juice transmittance

从图 2 可以看出, 不同温度对复合汁透光率影响不大, 温度在 $50^{\circ} \mathrm{C}$ 时, 透光率最大, 达到 $96 \%$ 以上, 复 合汁最适澄清温度为 $50^{\circ} \mathrm{C}$, 超过 $50^{\circ} \mathrm{C}$, 复合汁透光率缓慢降低。

\section{4.澄清时间对复合汁透光率的影响}

在壳聚糖用量 $1.6 \mathrm{~g} / \mathrm{L}, \mathrm{pH}=6$, 温度 $50^{\circ} \mathrm{C}$ 的条件下, 作用时间与复合汁透光率关系见图 3 。

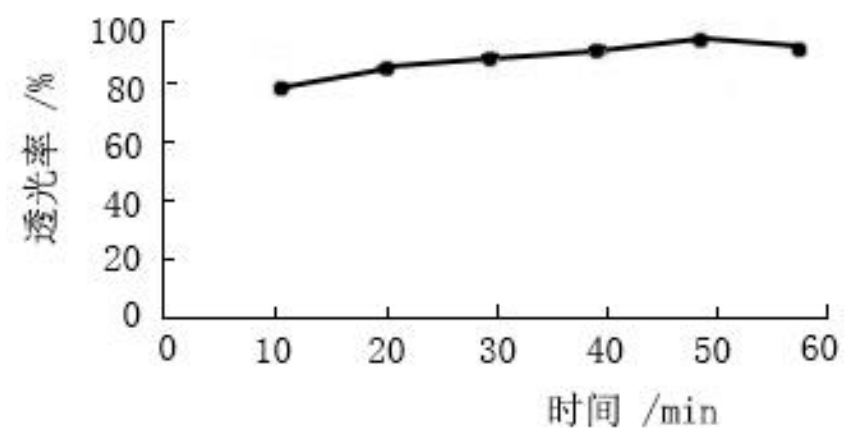

图 3 澄清时间对复合汁透光率的影响

Figure 3. The influence of clarification time on complex juice transmittance

由图3可知, 壳聚糖澄清复合汁在 $10 \mathrm{~min}$ 50min之间, 随作用时间的延长, 复合汁透光率略有增大, $50 \mathrm{~min}$ 透光率最高, 达到 $96.1 \%$ 。当澄清时间超过 $50 \mathrm{~min}$ 时, 随着澄清时间的加长, 透光率缓慢减小。

\section{$2.5 \mathrm{pH}$ 值对复合汁透光率的影响}

在壳聚糖用量 $1.6 \mathrm{~g} / \mathrm{L}$, 温度 $50^{\circ} \mathrm{C}$ 的条件下, 作用时间 $50 \mathrm{~min}$ 条件下, $\mathrm{pH}$ 值与复合汁透光率关系见图 4 。 


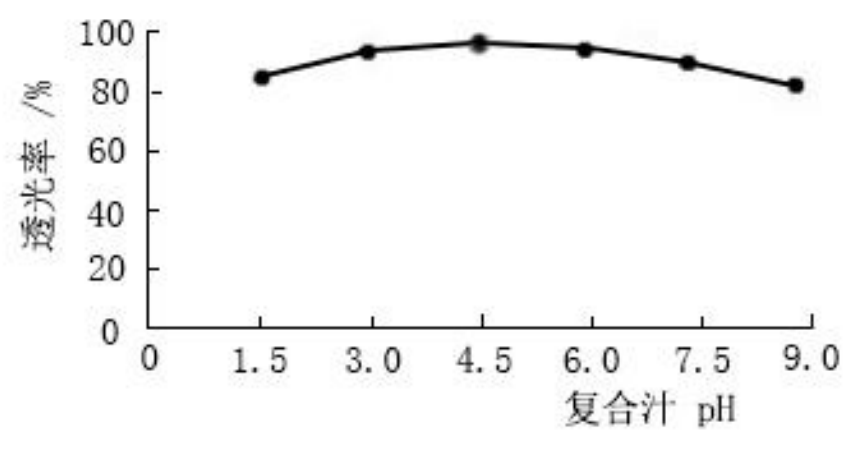

图 $4 \mathrm{pH}$ 值对复合汁透光率的影响

Figure 4. The influence of $\mathrm{pH}$ on complex juice transmittance

由图 4 可知, 在 $\mathrm{pH}<4.5$ 时, 随 $\mathrm{pH}$ 升高, 复合汁透光率逐渐增大, 但是, 增大幅度不大。 $\mathrm{pH}=4.5$ 时, 透光率达到 $96.4 \%$, 澄清效果最好。 $\mathrm{pH}>4.5$ 以后透光率降低。

\section{6 复合汁澄清最佳条件的确定}

根据以上复合汁澄清的单因素试验结果, 将壳聚糖澄清复合汁的正交试验设计成4因素3水平进行试 验。试验因素水平见表1。试验结果见表2。

\section{表 1 复合汁澄清的试验因素水平表}

Table 1 the experiment factors table of complex juice clarification

\begin{tabular}{ccccc}
\hline \multirow{2}{*}{ 水平 } & \multicolumn{2}{c}{ 因 } & 素 & \\
& 壳聚糖用量 $/ \mathrm{g} / \mathrm{L}$ & 温度 $/{ }^{\circ} \mathrm{C}$ & 澄清时间 $/ \mathrm{min}$ & $\mathrm{pH}$ \\
\hline 1 & 1.2 & 40 & 40 & 4.0 \\
2 & 1.6 & 50 & 50 & 5.0 \\
3 & 2.0 & 60 & 60 & 6.0 \\
\hline
\end{tabular}

表 2 复合汁澄清的正交试验及结果分析

Table 2 the orthogonal experiment and result analysis of complex juice clarification

\begin{tabular}{cccccc}
\hline \multirow{2}{*}{ 配方序号 } & \multicolumn{2}{c}{ 因 } & 素 & & 透光率/\% \\
& 壳聚糖用量 $/ \mathrm{g} / \mathrm{L}$ & 温度 $/{ }^{\circ} \mathrm{C}$ & 作用时间 $/ \mathrm{min}$ & $\mathrm{pH}$ & \\
\hline 1 & $1(1.2)$ & $1(40)$ & $1(40)$ & $1(4.0)$ & 88.5 \\
2 & 1 & $2(50)$ & $2(50)$ & $2(5.0)$ & 90.6 \\
3 & 1 & $3(60)$ & $3(60)$ & $3(6.0)$ & 91.4 \\
4 & $2(1.6)$ & 1 & 2 & 3 & 94.1 \\
5 & 2 & 2 & 3 & 1 & 96.1 \\
6 & 2 & 3 & 1 & 2 & 93.3 \\
7 & $3(2.0)$ & 1 & 3 & 2 & 90.6 \\
8 & 3 & 2 & 1 & 3 & 91.1 \\
9 & 3 & 3 & 2 & 1 & 89.3
\end{tabular}


Table 2, cont.

$\begin{array}{ccccc}\mathrm{K}_{1} & 270.5 & 273.2 & 272.9 & 273.9 \\ \mathrm{~K}_{2} & 283.5 & 277.8 & 274.0 & 274.5 \\ \mathrm{~K}_{3} & 271.0 & 275.3 & 278.1 & 276.6 \\ \mathrm{k}_{1} & 90.2 & 91.1 & 91.0 & 91.3 \\ \mathrm{k}_{2} & 94.5 & 92.6 & 91.3 & 91.5 \\ \mathrm{k}_{3} & 90.3 & 91.8 & 92.7 & 92.2 \\ \mathrm{R} & 4.3 & 1.5 & 1.7 & 0.9\end{array}$

由表2可知，对紫甘薯、大東、山楂复合汁澄清效果影响最大的因素是壳聚糖用量，其次是作用时间 和温度, 影响最小的是 $\mathrm{pH}$ 值。根据极差分析确定, 壳聚糖澄清该复合汁的最适工艺条件为: $\mathrm{pH}$ 值为6.0、 壳聚糖用量 $1.6 \mathrm{~g} / \mathrm{L}$ 、温度 $50^{\circ} \mathrm{C}$ 、澄清时间 $60 \mathrm{~min}$ 。

\section{3 结论}

采用壳聚糖对紫甘薯、大冭、山楂复合汁进行澄清时, 壳聚糖加入量、作用时间、温度和复合汁 $\mathrm{pH}$ 值 等因素对澄清效果都有影响。其中, 影响最大的因素是壳聚糖用量, 其次是温度和澄清时间, 影响最小的 因素是 $\mathrm{pH}$ 值。通过正交试验找出的最适工艺条件为: $\mathrm{pH}$ 值为6.0、壳聚糖用量 $1.6 \mathrm{~g} / \mathrm{L}$ 、温度 $50^{\circ} \mathrm{C}$ 、澄清时间 $60 \mathrm{~min}$ 。由此可见, 壳聚糖是一种有效的复合果汁澄清剂, 利用壳聚糖对紫甘薯、大杳、山楂复合汁进行 澄清, 澄清效果好, 可在饮料加工工业中应用。

\section{4 致谢}

基金项目名称：山东省科技厅星火计划项目(2012XH06004)。

\section{Acknowledgement}

Fund Project Name: Shandong Provincial Science and Technology Department Spark Program (2012XH06004).

\section{参考文献:}

[1] 孔芳, 高勇.紫甘薯的营养保健功能及研究进展[J].安徽农学通报,2013,(5);11-13

[2] 郭庆伟.大東的保健功能[J].农村新技术,2013,(2);43-43

[3] Chen J, Chan PH, Lam CT, Li Z, Lam KY, Yao P, Dong TT, Lin H, Lam H, Tsim KW. Fruit of Ziziphus jujuba (Jujube) at two stages of maturity: distinction by metabolic profiling and biological assessment. J Agric Food Chem. 2015 Jan 21;63(2):739-44.

[4] Ahmad A, Khan RM, Alkharfy KM. Effects of selected bioactive natural products on the vascular endothelium. J Cardiovasc Pharmacol. 2013 Aug;62(2):111-21.

[5] 付云霄,张桂,赵国群.壳聚糖在果汁澄清中的应用[J].食品工程,2011,(1);15-17

[6] 罗世江.影响壳聚糖絮凝法澄清效果因素浅析[J].中国医药指南,2012,10(12);455-457

[7] Ozge Tastan, Taner Baysal. Clarification of pomegranate juice with chitosan: Changes on quality characteristics during storage. Food Chemistry, Volume 180, 1 August 2015, Pages 211-218, ISSN 0308-8146

[8] Cesar LT, de Freitas Cabral M, Maia GA, et al. Effects of clarification on physicochemical characteristics, antioxidant capacity and quality attributes of açaí (Euterpe oleracea Mart.) juice. Journal of Food Science and Technology.

2014;51(11):3293-3300.

[9] Zhang H, Yun S, Song L, Zhang Y, Zhao Y. The preparation and characterization of chitin and chitosan under large-scale submerged fermentation level using shrimp by-products as substrate. Int J Biol Macromol. 2016 Dec 10;96:334-339. 
[10] 吴长青.壳聚糖在果汁澄清工艺上的应用[J].饮料工业，2001，(3)：9 11

\section{References:}

[1] Kong Fang, Gao Yong. Nutrition and Health Care Function of Purple Sweet Potato and its Research Progress [J].Anhui Agricultural Science Bulletin,2013,(5);11-13

[2] Guo Qingwei. Health Function of Chinese-Date [J].New Rural Technology,2013,(2);43-43

[3] Chen J, Chan PH, Lam CT, Li Z, Lam KY, Yao P, Dong TT, Lin H, Lam H, Tsim KW. Fruit of Ziziphus jujuba (Jujube) at two stages of maturity: distinction by metabolic profiling and biological assessment. J Agric Food Chem. 2015 Jan 21; 63(2):739-44.

[4] Ahmad A, Khan RM, Alkharfy KM. Effects of selected bioactive natural products on the vascular endothelium. J Cardiovasc Pharmacol. 2013 Aug; 62(2):111-21.

[5] Fu Yunxiao, Zhang Jia, Zhao Guoqun. Application of Chitosan in Clarification of Fruit Juices [J].Food Engineering,2011,(1);15-17

[6] Luo Shijiang. Clarification Effect of Chitosan Flocculation Factor [J].Guide of China Medicine,2012,10(12);455-457

[7] Ozge Tastan, Taner Baysal. Clarification of pomegranate juice with chitosan: Changes on quality characteristics during storage. Food Chemistry, Volume 180, 1 August 2015, Pages 211-218, ISSN 0308-8146

[8] Cesar LT, de Freitas Cabral M, Maia GA, et al. Effects of clarification on physicochemical characteristics, antioxidant capacity and quality attributes of açaí (Euterpe oleracea Mart.) juice. Journal of Food Science and Technology. 2014; 51 (11):3293-3300.

[9] Zhang H, Yun S, Song L, Zhang Y, Zhao Y. The preparation and characterization of chitin and chitosan under large-scale submerged fermentation level using shrimp by-products as substrate. Int J Biol Macromol. 2016 Dec 10; 96: 334-339.

[10]Wu Changqhing. Application of Chitosan in the Clarification Process [J].The Beverage Industry,2001,(3):9 11 